\title{
Serum ferritin in children with thalassaemia regularly transfused
}

\author{
E. A. LETSKY, F. MILLER, M. WORWOOD, AND D. M. FLYNN \\ From the Departments of Haematology of the Hospital for Sick Children, London, and the Welsh National \\ School of Medicine, Cardiff
}

SYNOPSIS A controlled trial of continuous chelation therapy in regularly transfused children with homozygous $\beta$-thalassaemia has been in progress at the Hospital for Sick Children since April 1966. In the sixth and seventh years of the trial the effect of this treatment on iron overload has been assessed by estimating serum ferritin levels and liver iron concentrations in both chelator-treated and control groups. When compared with non-chelated controls, results of both these estimations were invariably lower in the chelated group. However, all the results in both groups were very high, and fell within the ranges observed in untreated idiopathic haemochromatosis. A close correlation was found between serum ferritin levels and liver iron concentrations in these children, indicating that serum ferritin is a valuable alternative to liver iron concentration in the assessment of visceral iron overload, even when massive tissue siderosis is present.

Without blood transfusion the majority of patients with homozygous $\beta$-thalassaemia die from anaemia in early childhood. With regular transfusions, however, survival is prolonged into adolescence or early adult life (Weatherall and Clegg, 1972). The major problem in management, and the chief factor limiting life in these patients, then becomes that of iron overload, the excess iron being derived predominantly from transfused red blood cells.

Parenteral chelating agents have been used in thalassaemia in an attempt to promote mobilization and excretion of the excess iron but at present there is little information regarding their real value in this condition. A prospective trial of long-term chelation therapy in regularly transfused children with homozygous thalassaemia was started at the Hospital for Sick Children in 1966. During the sixth and seventh years of the trial serum ferritin levels were measured in both chelator-treated patients and in untreated controls. The results of these estimations have been compared with liver iron concentrations in the same patients, and serum ferritin levels have also been determined in other regularly transfused thalassaemic children not included in the trial. The results obtained provide information regarding the effects of chelation therapy and the value of the serum ferritin level as a measure of iron overload in thalassaemia.

Received for publication 20 May 1974.

\section{Materials and Methods}

\section{PATIENTS}

\section{The chelator trial}

Twenty regularly transfused children with homozygous $\beta$-thalassaemia were divided into two groups of 10 , matched as closely as possible for age, sex, transfusion requirements, and splenectomy status. One group received chelating agents and the other acted as controls. One child in the control group was lost to follow up almost immediately. Children allocated to the chelator-treated group received desferrioxamine and diethylene-triamine pentaacetate (DTPA) in addition to regular transfusions at intervals of four to six weeks aimed at maintaining haemoglobin levels above $8.5 \mathrm{~g} / \mathrm{dl}$. Full clinical details are given in the paper by Barry, Flynn, Letsky, and Risdon (1974).

In 1971-72 17 of the patients in the trial underwent liver biopsy for histological assessment and to determine the liver iron concentration. In addition, one girl in the control group died in April 1973 and the liver iron concentration was determined on a specimen of liver taken at necropsy. Serum ferritin levels were estimated in all 19 patients in the trial during 1972, and also in 15 young regularly transfused thalassaemic children not included in the trial during 1972-73. 


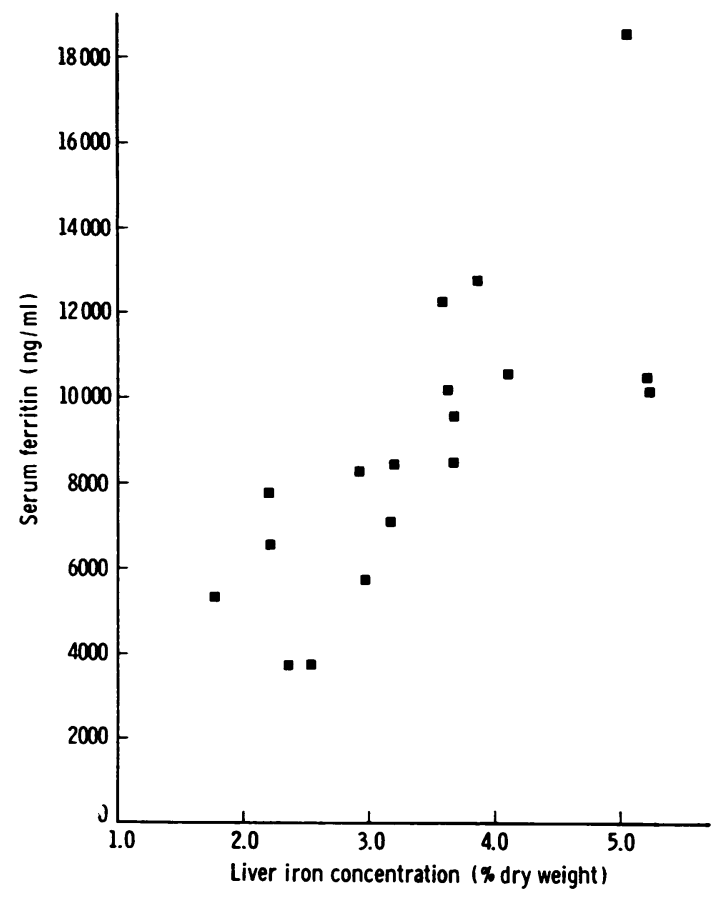

Fig 1 Relation between the serum ferritin levels and liver iron concentrations in 19 children included in the chelator trial $(r=0.75, \mathrm{P}<0.001)$.

Fig 2 Relation between the serum ferritin levels $(a)$ and liver iron concentrations $(b)$ and the number of units of blood transfused to children in the chelator trial. The liver iron levels were determined on biopsy specimens in 17 instances and on a necropsy specimen in one case. The ringed point (2a) represents the value obtained in a patient with terminal cardiac failure, ascites, and markedly raised transaminase levels due to hepatic venous congestion.

Fig 1
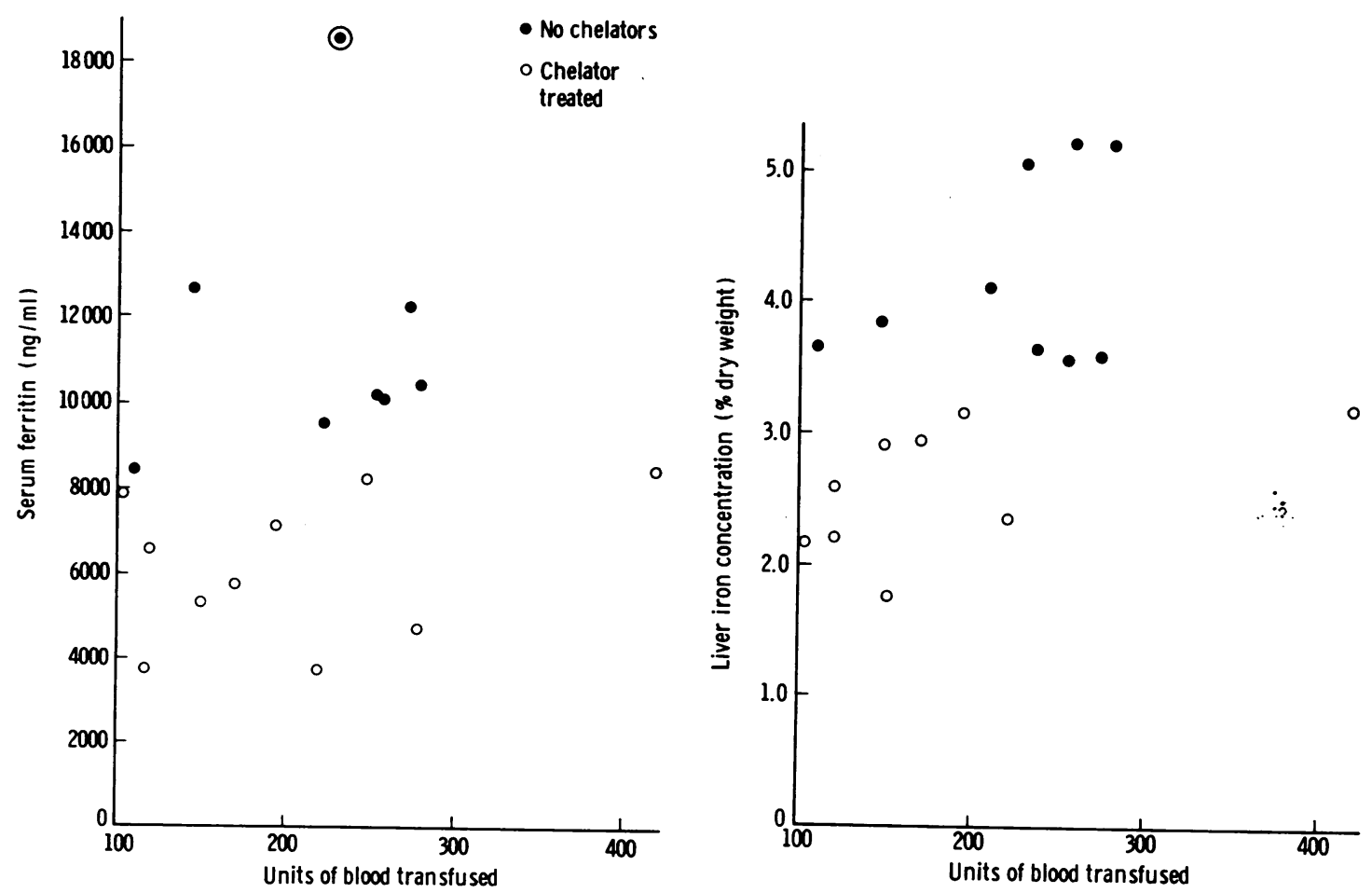

Fig 2a

Fig 2b 
LIVER IRON CONCENTRATIONS

Liver iron concentrations were determined by the method of Barry and Sherlock (1971). Specimens of liver were oven-dried and weighed to the nearest $0.01 \mathrm{mg}$ before chemical analysis.

\section{SERUM FERRITIN LEVELS}

Serum ferritin levels were estimated on venous blood samples by immunoradiometric assay using the method of Addison, Beamish, Hales, Hodgkins, Jacobs, and Llewellin (1972).

\section{Results}

There was a highly significant correlation (fig 1) between the liver iron concentrations and the serum

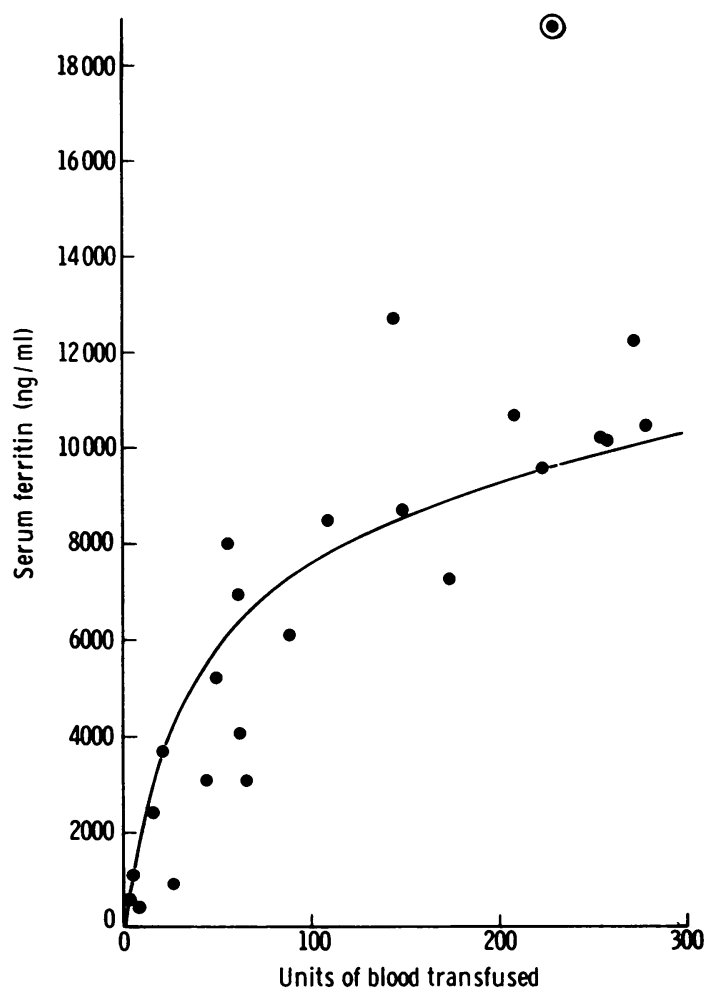

Fig 3 Comparison between the serum ferritin levels and the number of units of blood received in 24 regularly transfused thalassaemic children, none of whom had been treated with chelating agents. Nine of these patients were from the control group in the trial $(r=0 \cdot 88)$.

The regression line is derived from the equation: $y=5659 \log x-3767$.

The ringed point (see also fig 2a) was excluded from the statistical analysis (see text). ferritin levels in the patients included in the trial. Liver iron and serum ferritin values in all members of both groups were, however, very high and fell within the range seen in untreated haemochromatosis. In figs $2 \mathrm{a}$ and $2 \mathrm{~b}$ serum ferritin levels and liver iron concentrations are plotted against the number of units of blood transfused. Both measurements were invariably lower in the chelator-treated group thàn in the controls. Figure 3 compares the serum ferritin levels with the amount of blood received in a number of regularly transfused thalassaemics, none of whom had been given chelating agents. This shows that an initial steep rise in the serum ferritin levels occurred during the time when the first 50-100 units of blood were given, after which further transfusion led to a proportionately smaller increase.

\section{Discussion}

The definitive method of measuring the body iron stores is by repeated venesection to the point of anaemia, that is, by measurement of the mobilizable storage iron (Haskins, Stevens, Finch, and Finch 1952). This is obviously not feasible in patients with thalassaemia, in whom an alternative method of assessing iron stores is necessary. Two such techniques are available, namely, measurement of liver iron concentration and DTPA-chelatable body iron; the results obtained in these alternative methods have been shown to be closely correlated both with each other and with mobilizable storage iron in a variety of iron-loading disorders including splenectomized and non-splenectomized patients with thalassaemia (Barry, 1973; Barry, 1974). Thus, within acceptable limits, liver iron concentration reflects total body storage iron. A high degree of correlation has been demonstrated between serum ferritin concentration and storage iron measured by quantitative phlebotomy in normal subjects (Walters, Miller, and Worwood, 1973). The highly significant correlation we have demonstrated between serum ferritin levels and liver iron concentrations shows that serum ferritin is a valid alternative measurement of the iron stores, even at the very high levels found in our patients with thalassaemia. As an estimate of the total storage iron it has the obvious advantages over the liver iron concentration that a liver biopsy is not required and that serial determinations can easily be performed.

Our results in the children included in the trial show clearly that serum ferritin and liver iron concentrations are lower in the chelator-treated children than in the controls. It is well recognized that there is often a marked individual variation in the severity of thalassaemia but it is unlikely that this accounts for the differences observed be- 
tween the two groups. Both the severity of the disease and the iron load received are reflected in the transfusion requirements, yet the serum ferritin levels and the liver iron concentrations were invariably lower in the chelator-treated subjects even when these were correlated with the number of units of blood received. However, both estimations were very high in all children included in the trial, indicating total body iron loads well within and above the range seen in untreated haemochromatosis (Jacobs, Miller, Worwood, Beamish, and Wardrop, 1972; Barry, 1974). A single exceptionally high ferritin level was observed in a patient with terminal congestive cardiac failure at a time when the serum transaminase levels were markedly elevated due to hepatic congestion. Since serum ferritin levels have been shown to fluctuate in parallel with serum transaminase levels in patients with liver disease (Prieto, Barry, and Sherlock, 1974) this value was probably not a true reflection of the total storage iron in this patient.

When the serum ferritin levels in regularly transfused thalassaemic children who had not received chelating agents were correlated with the amount of blood transfused it was apparent that the first 50-100 units produced a steep rise in serum ferritin after which the rate of increase was less marked. One explanation for the fall in the rate of increase of serum ferritin could be the conversion of ferritin to insoluble haemosiderin which occurs at high storage levels but serial estimations of liver iron concentrations in the control group of the chelator trial gave closely similar results (Barry et al, 1974). The faliing off in the rate of liver iron accumulation in massively iron-loaded subjects is open to several interpretations, one of which would be that it is due to an increase in the proportion of iron deposited in sites other than the liver. This hypothesis would not, however, explain the similar falling off in serum ferritin levels, since these are a measure of the total amount of iron stored in the body. An alternative suggestion is that the decrease in storage iron relative to the transfused load observed in heavily ironloaded patients is due to the onset of spontaneous iron excretion. This view is supported by a number of reports (Howell and Wyatt, 1953; Cappell, Hutchison, and Jowett, 1957; Witzleben and Wyatt, 1961) indicating that estimates of cadaver iron in heavily transfused subjects fall far short of the total amount of iron received in life.

The similarity between the liver iron concentrations and serum ferritin levels in our massively transfused subjects and the apparent falling off in the rate of accumulation of iron indicated by both these estimations suggest that serum ferritin is a useful measure of totai body iron stores at all stages of transfusion siderosis in thalassaemia.

We wish to thank Dr M. Barry who estimated all the liver iron concentrations.

F.M. is supported by a grant from the Leukaemia Research Fund.

\section{References}

Addison, G. M., Beamish, M. R., Hales, C. N., Hodgkins, M., Jacobs, A., and Llewellin, P. (1972). An immunoradiometric assay for ferritin in the serum of normal subjects and patients with iron deficiency and iron overload. J. clin. Path., 25, 326-329.

Barry, M. (1973). Iron overload: clinical aspects evaluation and treatment. Clin. Haemat., 2, no. 2.

Barry, M. (1974). Liver iron concentration, stainable iron, and total body storage iron. Gut, 15, 411-415.

Barry, M., Flynn, D. M., Letsky, E. A., and Risdon, R. A. (1974). Long-term chelation therapy in thalassaemia major: the effect on liver iron concentration, liver histology and clinical progress. Brit. med. J., 2, 16.

Cappell, D. F., Hutchison, H. E., and Jowett, M. (1957). Transfusional siderosis: the effects of excessive iron deposits on the tissues. . J. Path. Bact., 74, 245-264.

Haskins, D., Stevens, A. R., Jr., Finch, S., and Finch, C. A. (1952). Iron metabolism: iron stores in man as measured by phlebotomy. J. clin. Invest., 31, 543-547.

Howell, J., and Wyatt, J. P. (1953). Development of pigmentary cirrhosis in Codey's anemia. Arch. Path., 55, 423-431.

Jacobs, A., Miller, F., Worwood, M., Beamish, M. R., and Wardrop, C. A. (1972). Ferritin in the serum of normal subjects and patients with iron deficiency and iron overload. Brit. med. J., 4, 206-211.

Prieto, J., Barry, M., and Sherlock, S. (1974). Serum ferritin in patients with iron overload and with acute and chronic liver disease. (Abstr.) Gut, 15, 343.

Walters, G. O., Miller, F. M., and Worwood, M. (1973). Serum ferritin concentration and iron stores in normal subjects. $J$. clin. Path., 26, 770-772.

Weatherall, D. J., and Clegg, J. B. (1972). The Thalassaemia Syndromes, 2nd col. Blackwell, Oxford.

Witzleben, C. L., and Wyatt, J. P. (1961). The effect of long survival on the pathology of thalassaemia major. J. Path. Bact., 82, $1-12$. 